\title{
A Review of Nasal Polyps
}

\author{
Eyad Zein Aldean
}

Specialized Medical Care hospital, UAE

\author{
Corresponding author \\ Dr. Eyad Zein Aldean MD, MSc, UBMS ORL-HNS, Specialized Medical \\ Care Hospital, UAE. E-mail: dreyadenthns@gmail.com
}

Submitted: 04 Nov 2019; Accepted: 16 Nov 2019; Published: 29 Nov 2019

\begin{abstract}
Nasal polyps (NP) are one of the most common inflammatory lesions of the nose, affecting up to 4\% of the population. Their etiology remains unclear, but they are known to have associations with allergy, asthma, infection, cystic fibrosis, and aspirin sensitivity. However, the underlying mechanisms interlinking these pathologic conditions to NP formation remain unclear. Also strong genetic factors are implicated in the pathogenesis of $N$, but genetic and molecular alterations required for its development and progression are still unclear. They present with nasal obstruction, anosmia, rhinorrhea, post nasal drip, and less commonly facial pain.

Management of polyposis involves a combination of medical therapy and surgery. There is good evidence for the use of corticosteroids (systemic and topical) both as primary treatment and as postoperative prophylaxis against recurrence. Surgical treatment has been refined significantly over the past twenty years with the advent of endoscopic sinus surgery and, in general, is reserved for cases refractory to medical treatment. Recurrence of the polyposis is common with severe disease recurring in up to ten percent of patients. In this talk I will present the newer treatment options available for better control and possibly cure of the disease.
\end{abstract}

Keywords: Nasal polyps, Disease Management, Surgical Procedures

\section{Introduction}

Nasal polyposis (NP) is one of the most common mass lesions of the nose and was first described 4000 years ago in ancient Egypt. Hippocrates was the first who named "polyp" to the tissue mass with a bell-clapper shape in the nasal cavity the prevalence of NP is reported from 0.2 to $4.3 \%$ worldwide, with a ratio of 2-3: 1 between male and female individuals. In children, NP is relatively rare and has a close relationship with asthma and cystic fibrosis despite its easy diagnosis, it is a challenge for otorhinologists, because of its poorly understood etiopathogenesis, poor impact of therapeutic intervention and frequent recurrences. It is a multifactorial condition which is often associated with many diseases and pathogenic disorders, such as allergy, infection, allergic fungal sinusitis, cystic fibrosis, asthma, and aspirin intolerance. However, the underlying mechanisms interlinking these pathologic conditions to NP formation remain unclear. Although the exact etiology of nasal polyposis is still not revealed, insights in the pathogenesis have largely expanded over the last years. Increasing insights in the pathophysiology of nasal polyposis opens perspective for new pharmacological treatment options, with eosinophilic inflammation, $\mathrm{IgE}$, fungi and Staphylococcus aurous as potential targets.

\section{Pathogenesis}

NPs are outgrowths of nasal mucosa which are smooth, semi translucent, gelatinous and pale, mainly situated in the middle meatus, originating from mucous membrane of the osteomeatal complex, probably because of release of pro inflammatory cytokines from epithelial cells as a result of contact between two surfaces of mucosa at this narrow region. Air turbulence and pressure differential may also have an influence. Various other important factors like genetic factors, bacteria, fungi, biofilm formation, etc. have been implicated, and have been discussed in subsequent paragraphs. Histo-morphological characterization of polyp tissue reveals frequent epithelial damage, a thickened basement membrane, and edematous to sometimes fibrotic stromal tissue, with a reduced number of vessels and glands, but virtually no neural structure. Polyps show an increased number of mast cells, eosinophils, T lymphocytes, cytokines, chemokines, interleukins, TNF- $\alpha$ and adhesion molecules (Figure 1).

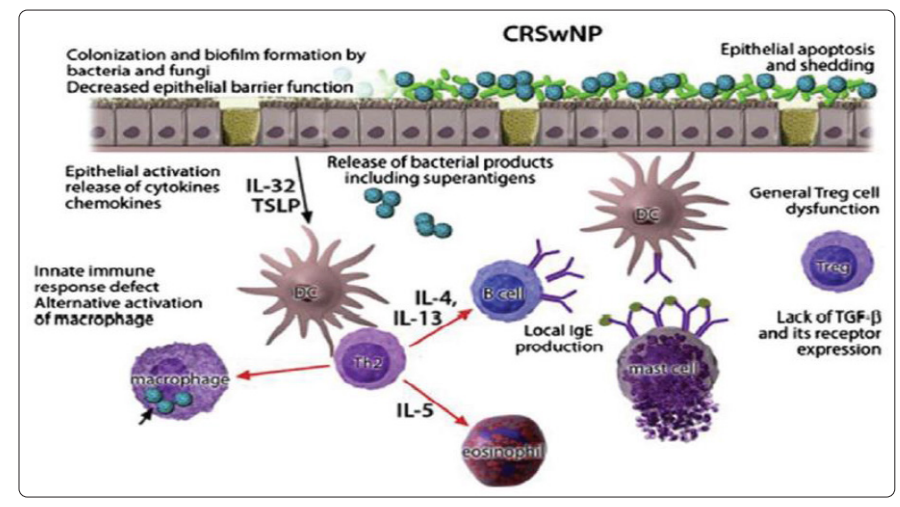

Figure 1 
Role of Genetic Factors in Pathogenesis

During the past two decades, many studies have been performed to determine differential gene expression profiles between NP and normal nasal tissues, in order to identify susceptible genes that are associated with NP-related traits. A number of genetic association studies found a significant correlation between certain human leukocyte antigen (HLA) alleles and NP. The risk of developing NP can be as high as 5.53 times in subjects with HLA-DQA $1 * 0201$ DQB1*0201 haplotype [1]. The development and persistence of mucosal inflammation in NPs have been reported to be associated with numerous genes and potential single nucleotide polymorphisms. A recent study showed that in NP tissues, 192 genes were up regulated by at least twofold, and 156 genes were down regulated by at least $50 \%$ in NP tissues as compared to sphenoid sinuses mucosa [2]. It has also been postulated that an abnormal mucosal immune response underlies disease pathogenesis [3]. There are a number of genes which are involved in epithelial barrier maintenance and repair in the inflammatory state of NP. For example, carbonic anhydrase (CA) is a zinc metalloenzyme that participate in the biological processes of various fluid transporting epithelia, including ion and water transport. A decreased expression level of CA was found to be associated with impaired electrolyte and water transport across the epithelial cell, which will result in edema of NP tissue [4]. Identifying the causal genes and variants in NP is important to the path towards improved prevention, diagnosis and treatment of NPs.

\section{Role of Fungus}

Among the possible etiologies, fungi have gained wide attention in recent years. Though fungal particles are present in sinonasal mucosa of healthy subjects too, but they act as antigens in mucosa of sensitized individuals, resulting in recruitment of inflammatory cells - namely eosinophils - and release of major basic protein (MBP), which finally causes mucosal damage and super infection by migration of other inflammatory cells into that location [5]. This fungal antigen is derived from the germinating fungal spores and hyphae. This inflammatory reaction is different from the one seen in response to a fungus ball which is more of an irritative inflammation, like a foreign body reaction, i.e., giant cells, and not an eosinophilic inflammation, which is present in nasal polyposis [6]. Aspergillus and Alternaria are commonest fungi species implicated in the pathogenesis of nasal polyposis [7].

\section{Role of Biofilms}

Microorganisms like bacteria and fungi exist in two main forms in the sinonasal cavities: as free-floating planktonic replicating cells and in biofilms. Biofilms are defined as organized communities of collaborating microorganisms that are attached to an inert or living surface contained in a self-produced polymeric matrix primarily composed of exopolysaccharides, nucleic acids, and proteins [8]. The structural nature of biofilms and the characteristics of sessile cells produce resistance against antimicrobial agents, resulting in an environment that affords protection against adverse conditions and the host's defenses [9]. The bacteria in these biofilms, while protected from host defenses and antibiotics, actively metabolize and produce endotoxins and other virulence factors. This may perpetuate an inflammatory host response, even in the absence of culturable planktonic bacteria and lead to chronic inflammation [10]. Traditional antimicrobial treatments that target single microbial cells within biofilms will never be able to eliminate them. Therefore, antibiofilm therapies that target the entire biofilm as a complex multicellular organism or prevent unique, biofilm-specific processes are needed to fight biofilm infections.

\section{Management of Sinonasal Polyposis}

Therapy for NP involves a combination of observation, medical, and surgical treatments depending on individual case assessment. The aims of treatment are to eliminate or significantly reduce the size of the NP resulting in relief of nasal obstruction, improvement in sinus drainage, restoration of olfaction and taste. Treatment of Surgical procedures alone is insufficient to treat the underlying inflammation of the nasal mucosa. Supplementary medical treatment is always necessary to prevent recurrence.

\section{Medical Management}

Intranasal glucocorticoids constitute presently the best treatment of NP. They decrease polyp size, improve nasal airway patency, improve symptoms of rhinitis like rhinorrhea, sneezing and nasal blockage, delay the recurrence of polyps after surgery and postpone the need for a new surgery [11]. The unusually wide range of GC actions can be explained by $\mathrm{GC}$ receptors present in three cell compartments: nucleus, cytoplasm, and plasma membrane. Both topical and systemic glucocorticoids may affect the eosinophil function by both directly reducing eosinophil viability and function or indirectly reducing the secretion of chemotactic cytokines by nasal mucosa and polyp epithelial cells [12]. Systemic steroids are reserved for advanced or refractory cases particularly when allergy is present and it results in relatively rapid short-term dramatic improvement, nasal symptoms and endoscopic findings (medical polypectomy). Simple saline nasal douching to help cleanse the nose prior to topical medications is beneficial as it improves nasal mucociliary clearance. Corticosteroids should be used with caution in 'at-risk groups' particularly patients with diabetes, uncontrolled hypertension, and peptic ulcer disease.

\section{Role of Leukotrienes Antagonists}

Leukotrienes (LTs) and prostaglandins are products of arachidonic acid metabolism, and are key mediators in acute and chronic inflammatory diseases of the airways. Leukotriene levels have been shown to be elevated in patients with sinonasal polyposis and sinusitis. Recent studies have shown, an objective alleviation, or at least stabilization, of sinonasal polyposis after use of short-term oral corticosteroid therapy combined with the LT synthesis inhibitor zileuton or the LT receptor antagonist zafirlukast and montelukast as maintenance therapy [13]. These improvements are probably based on the control of NP inflammation and possibly of polyp growth. So short-term oral corticosteroid therapy combined with montelukast in a daily dosage of $10 \mathrm{mg}$ as maintenance therapy in controlling symptoms of severe sinonasal polyposis has been proven very effective. Also, an additional 3 months of montelukast therapy combined with intranasal and inhaled corticosteroids produces subjective and objective improvements in nasal symptoms and function as well as significant improvements in lung function in patients with nasal polyposis [14].

\section{Role of S. aureus and Concept of Superantigens}

Evidence accumulates that $\mathrm{S}$. aureus colonizes chronic rhinosinusitis with, but not without polyps, with significantly increased prevalence. The germs release enterotoxins, which act as superantigens and induce a topical multiclonal $\mathrm{IgE}$ formation as well as a severe, possibly steroidinsensitive eosinophilic inflammation [15]. Recently, $\mathrm{S}$. aureus could be demonstrated to reside intraepithelially, and potentially to release superantigens into the tissue from within the 
epithelial cells. An immune defect, either in the innate or adaptive immunity, might be responsible for this phenomenon. Folliclelike structures and lymphocyte accumulations, specifically binding enterotoxins, can be found within the polyp tissues, giving rise to local IgE formation. The superantigen-induced immune response also leads to a modulation of the severity of the eosinophilic inflammation, and may be linked to lower airway co-morbidity in polyp patients. IgE antibodies to enterotoxins can be found in the majority of aspirin-sensitive polyp tissues, associated with a substantial increase in eosinophilic cationic protein (ECP) and IL-5 [16].

\section{Role of Antibiotics}

Based on the concept of S. aureus intraepithelial colonization, studies have been done to support the use of antibiotics along with corticosteroids to treat patients with NP. Recent studies have shown that oral doxycycline $(200 \mathrm{mg}$ on the first day, followed by 100 mg once daily) for 20 days has shown a significantly decreased NP size, reduced levels of myeloperoxidase, ECP, and matrix metalloproteinase 9 in nasal secretions [17].

\section{Role of Anti IgE Therapy}

Based on the concept of S. aureus derived enterotoxins acting as super antigens, massive IgE formation takes place within the airways. Because of the multiclonality, a range of allergens could possibly maintain a constant degranulation of mast cells present in the polyp tissue, which may contribute to disease severity. Omalizumab counteracts these interactions by reducing serum levels of free $\mathrm{IgE}$. Therapy targeted at IgE also interferes with its binding to the low-affinity receptors inhibiting the amplification of the Th2-type response [18]. The high costs of treatment with omalizumab, the high frequency of NP, as well as the current lack of data concerning safety in long-term application of omalizumab has to be borne in mind and further studies have to be conducted [19].

\section{Role of CMC Foam}

Recurrence of nasal polyposis after endoscopic sinus surgery can be difficult to manage. Topical steroid sprays and irrigations may not provide adequate treatment and systemic steroid therapy is limited by side effects. Steroid-infused carboxymethylcellulose (CMC) foam as a treatment for recurrence of chronic rhinosinusitis with nasal polyposis after endoscopic sinus surgery has been tried. Four milliliter of CMC foam hydrated with triamcinolone, $40 \mathrm{mg}$ / $\mathrm{ml}$ is placed endoscopically into the ethmoid cavities bilaterally. Statistically significant endoscopic results were obtained regarding improvement in symptoms and endoscopic findings in patients with recurrent sinonasal polyposis after endoscopic sinus surgery [20].

\section{Surgical Management}

Surgical therapy is reserved for cases refractory to medical treatment. In general, patients are treated medically in the primary care setting before consideration of surgical procedures by an otolaryngologist. Endoscopic sinus surgery is now the mainstay of treatment for NP, though no single surgical technique has proved to be entirely curative and the recurrence rate is around 5-10\% [2124]. Concurrent use of micro debrider enables accurate removal of NP whilst preserving normal anatomical structures such as the turbinates. Thorough knowledge of sinus anatomy, combined with preoperative imaging helps to avoid major complications such as blindness and CSF rhinorrhoea. Also the use of computer-aided surgery (CAS) technology, which allows a direct comparison of the intraoperative anatomy with preoperative imaging information furthers the operative accuracy. After a registration and calibration process, the surgeon may point to a specific structure with the CAS instrument and then view the location of the instrument tip on the CT image $[25,26]$. The use of CAS systems may allow for more precise dissections and greater rates of sinus patency outcomes and fewer complications [27]. It is important postoperatively to regularly douche the nasal cavity with saline to prevent crusting and adhesions [28]. Topical intranasal steroids are also a routine part of after surgery care to prevent recurrence [29].

\section{Conclusion}

With improvement in the management protocols and further research, nasal polyposis would no longer remain a challenge for otorhinolaryngologists. Future studies are needed to identify the key factors underlying the development or formation of NP and to investigate the interactions between genetic, local and environmental factors that influence the complex traits of this disease. Identifying the causal factors and variants in NP is important to the path towards improved prevention, diagnosis and treatment of NPs.

\section{References}

1. Fajardo-Dolci G, Solorio-Abreu J, Romero-Alvarez JC, Zavaleta-Villa B, Cerezo-Camacho O, et al. (2006) DQA1 and DQB1 association and nasal polyposis. Otolaryngol Head Neck Surg 135: 243-247.

2. Liu Z, Kim J, Sypek JP, Wang IM, Horton H, et al. (2004) Gene expression profiles in human nasal polyp tissues studied by means of DNA microarray. J Allergy Clin Immunol 114: 783-790.

3. Ramanathan M, Jr, Lee WK, Spannhake EW, Lane AP (2008) Th2 cytokines associated with chronic rhinosinusitis with polyps down-regulate the antimicrobial immune function of human sinonasal epithelial cells. Am J Rhinol 22: 115-121.

4. Kim TH, Lee HM, Lee SH, Kim HK, Lee JH, Oh KH (2008) Down-regulation of carbonic anhydrase isoenzymes in nasal polyps. Laryngoscope 118: 1856-1861.

5. Davis LJ, Kita H (2004) Pathogenesis of chronic rhinosinusitis: role of airborne fungi and bacteria. Immunol Allergy Clin North Am 24: 59-73.

6. Yoshinari Inoue, Yoshinori Matsuwaki, Seung-Heon Shin, Jens U. Ponikau and Hirohito Kita, et al. (2005) Nonpathogenic, environmental fungi induce activation and degranulation of human eosinophils. J Immunol 175: 5439-5447.

7. Weschta M, Rimek D, Formanek M, Polzehl D, Riechelmann H (2003) Local production of Aspergillus fumigatus specific immunoglobulin E in nasal polyps. Laryngoscope 113: 17981802 .

8. Gilbert P, Maira-Litran T, McBain AJ, Rickard AH, Whyte FW (2002) The physiology and collective recalcitrance of microbial biofilm communities. Adv Microb Physiol 46: 202-256.

9. Foreman A, Psaltis AJ, Tan LW, Wormald PJ (2009) Characterization of bacterial and fungal biofilms in chronic rhino-sinusitis. Am J Rhinol Allergy 23: 556-561.

10. Kilty SJ, Desrosiers MY (2008) The role of bacterial biofilms and the pathophysiology of chronic rhinosinusitis. Curr Allergy Asthma Rep 8: 227-233.

11. Pujols L, Alobid I, Benitez P, Martinez-Antón A, Roca-Ferrer J, et al. (2008) Regulation of glucocorticoid receptor in nasal polyps by systemic and intranasal glucocorticoids. Allergy 63: 1377-1386. 
12. Grzanka A, Misiołek M, Golusiński W, Jarząb J (2011) Molecular mechanisms of glucocorticoids action: implications for treatment of rhinosinusitis and nasal polyposis. Eur Arch Otorhinolaryngol 268: 247-253.

13. Modrzynski M, Zavisza E, Rapiejko P (2002) Zafirlukast in treatment of nasal polyposis in patients with aspirin intolerant bronchial asthma-preliminary report. Pol Merkur Lekarski 12: 224-227.

14. Zhang N, Gevaert P, Van Zele T, Perez-Novo C, Patou J, et al. (2005) An update on the impact of Staphylococcus aureus enterotoxins in chronic sinusitis with nasal polyposis. Rhinology 43: 162-168.

15. Kutting B, Nieschalk M, Brehler K (2000) A new concept for treatment of sinonasal polyposis. Allergy 55: 1091-1092.

16. Zhang N, Gevaert P, van Zele T, Perez-Novo C, Patou J, et al. (2005) An update on the impact of Staphylococcus aureus enterotoxins in chronic sinusitis with nasal polyposis. Rhinology. 43: 162-168.

17. Van Zele T, Gevaert P, Holtappels G, Beule A, Wormald PJ, et al. (2010) Oral steroids and doxycycline: two different approaches to treat nasal polyps. J Allergy Clin Immunol 125: 1069-1076.

18. Incorvaia $\mathrm{C}$, Mauro M, Riario-Sforza GG, Frati F, Tarantini F, et al. (2008) Current and future applications of the anti-IgE antibody omalizumab. Biologics 2: 67-73

19. Holgate ST, Djukanovid R, Casale T, Bousquet J (2005) Antiimmunoglobulin $\mathrm{E}$ treatment with omalizumab in allergic diseases: an update on anti-inflammatory activity and clinical efficacy. Clin Exp Allergy 35: 408-416.
20. Pletcher SD, Goldberg AN (2010) Treatment of recurrent sinonasal polyposis with steroid-infused carboxymethylcellulose foam. Am J Rhinol Allergy 24: 451-453.

21. Telmesani LM (2009) Prevalence of allergic fungal sinusitis among patients with nasal polyps. Ann Saudi Med 29: 212-214.

22. Poetker DM, Mendolia-Loffredo S, Smith TL (2007) Outcomes of endoscopic sinus surgery for chronic rhinosinusitis associated with sinonasal polyposis. Am J Rhinol 21: 84-88.

23. Bhattacharyya $N$ (2007) Influence of polyps on outcomes after endoscopic sinus surgery. Laryngoscope 117: 1834-1838.

24. Wynn R, Har-El G (2007) Recurrence rates after endoscopic sinus surgery for massive sinus polyposis. Laryngoscope 114: 811-813.

25. Anon JB (1998) Computer-aided endoscopic sinus surgery. Laryngoscope 108: 949-961.

26. Olson G, Citardi MJ (2000) Image-guided functional endoscopic sinus surgery. Otolaryngol Head Neck Surg 123: 188-194.

27. Al-Swiahb JN, Al-Dousary SH (2010) Computer-aided endoscopic sinus surgery: a retrospective comparative study. Ann Saudi Med 30: 149-152.

28. Talbot AR, Herr TM, Parsons DS (1997) Mucocilliary clearance and buffered hypertonic saline solution. Laryngoscope 107: 500-503.

29. Newton RJ, Ah-See KW (2008) A review of nasal polyposis. Ther Clin Risk Manag 4: 507-512.

Citation: Eyad Zein Aldean (2019) A Review of Nasal Polyps. Med Clin Res 4(11): 1-4.

Copyright: (02019 Eyad Zein Aldean. This is an open-access article distributed under the terms of the Creative Commons Attribution License, which permits unrestricted use, distribution, and reproduction in any medium, provided the original author and source are credited. 